\title{
LIQUIDEZ Y EL FLUJO DE GAJA LIBRE, VALORACIÓN DE LAS EMPRESAS DE CERÁMICA DE GÚ́CUTA
}

\section{LIQUIDITY AND THE FREE CASH FLOW, VALUATION OF THE CERAMIC COMPANIES FROM CUCUTA}

\author{
Naydú Jácome Castilla ${ }^{1}$ \\ Magda Sepúlveda Angarita ${ }^{2}$
}

\section{RESUMEN}

El presente capítulo es el resultado de un proyecto de investigación cuya finalidad fue realizar un análisis de la liquidez y el flujo de caja libre como método de valoración de las empresas de cerámica de Cúcuta, siendo el sector cerámico en Norte de Santander uno de los generadores de mayor ingresos en términos de Producto Interno Bruto y de generación de empleo en el departamento. Desde la perspectiva de generación de valor, se revisaron las cifras de los estados financieros, analizando el comportamiento de las cuentas por cobrar, de los inventarios, y cómo estas influyeron en el flujo de caja libre de las empresas de cerámica de Cúcuta. Se realizó una investigación descriptiva y correlacional. Se tomó como muestra intencional la empresa Cerámica Italia por ser la organización de fabricación de productos minerales no metálicos que genera mayores ingresos en el

1 Universidad Simón Bolívar. Cúcuta, Colombia. Universidad Francisco de Paula Santander Ocaña. njjacomec@ufpso.edu.co

n.jacome@unisimonbolivar.edu.co - https://orcid.org/0000-0001-9241-0522

2 Universidad Francisco de Paula Santander. Cúcuta, Colombia.

magdazarelasa@ufps.edu.co 
departamento. Se caracterizaron las cuentas por cobrar, los inventarios, y el flujo de caja libre y mediante la aplicación de relaciones del coeficiente de correlación se determinaron la relación, la gestión del efectivo de la empresa, la liquidez, el flujo de caja libre y la generación de valor.

Palabras clave: Cuentas por cobrar, Inventarios, Flujo de caja libre y Liquidez.

\section{ABSTRACT}

This book chapter was aimed to make an analysis of liquidity and free cash flow as a valuation method for Cucuta ceramic companies with the ceramic sector in Norte de Santander one of the generators with the highest incomes in terms of Gross Domestic Product and employment generation in the department. From the perspective of generating value, the financial statement figures were reviewed, analyzing the behavior of accounts receivable and inventories, and how they influenced the free cash flow of Cucuta ceramic companies. A descriptive and correlational research was carried out. The Ceramic Company Italia was taken as an intentional sample because it is the organization of manufacture of nonmetallic mineral products that generates greater income in the department. Accounts receivable, inventories, and free cash flow were characterized and by applying correlation coefficient relationships were determined the relationship of cash management, liquidity, free cash flow and the generation of value.

Keywords: Accounts receivable, Inventories, free cash flow and Liquidity. 


\section{INTRODUCCIÓN}

El presente estudio incluye un análisis de la estructura financiera de las empresas de cerámica de Cúcuta en los años 2005 a 2015, desagregando el comportamiento de las cuentas por cobrar a clientes, los inventarios y el flujo de caja libre, se caracterizaron estos indicadores a partir de los estados financieros de la empresa Cerámica Italia que desarrolla el objeto social de fabricar productos minerales no metálicos y mediante un análisis de correlación se estableció el grado de asociación de las variables estudiadas para determinar la generación o destrucción de valor de las empresas de cerámica de Cúcuta.

A partir de los resultados se establece la matriz de Debilidades, Oportunidades, Fortalezas y Amenazas (DOFA) financiera y se plantean recomendaciones para la mejora de los procesos financieros.

\section{ESTADO DEL ARTE}

La fundamentación teórica del proyecto se enmarca en los postulados de liquidez, rentabilidad, administración del capital de trabajo, flujo de caja libre, la gerencia del valor y la sostenibilidad financiera, es así como, mediante las teorías financieras se realizó un análisis del comportamiento en cuanto a la liquidez y generación de valor de las empresas de cerámica de Cúcuta, correlacionando las variables financieras de liquidez, flujo de caja libre y el análisis contable de las cuentas por cobrar y los inventarios.

En este sentido, al observar la importancia del sector en el país, se encontró que el proceso de producción y su cadena productiva contribuye a un importante desarrollo económico e industrial, que permite la 
generación de recursos económicos y financieros en índices de Producto Interno Bruto y (PIB) la creación de empleos.

La argumentación básica teórica de la cadena productiva de la cerámica incluye los productos resultantes de la fabricación industrial de artículos de barro, loza y porcelana. Según el (DNP) ( s.f.), “Los productos de esta cadena hacen parte del sector productor de productos no metálicos, al igual que los productos de vidrio y cemento, y se encuentran agrupados en el sector CIUU 361" (p.381).

Por otra parte, la importancia de la cerámica dentro de la industria radica en su estrecho vínculo con el sector de la construcción, proporcionando productos empleados en el revestimiento de pisos y paredes, así como productos de porcelana sanitaria. De esta forma se constituye la principal fuente de materia prima en el sector minero y el sector químico. La elaboración industrial de productos cerámicos requiere de tres componentes básicos: plásticos, que aportan maleabilidad; no plásticos, evitan deformaciones o grietas; y fundentes, que rebajan la temperatura de vitrificación de la cerámica haciéndola densificar más rápidamente.

A diferencia de lo que sucede con los demás sectores pertenecientes a los minerales no metálicos (cemento y vidrio), el sector de la cerámica incorpora mayor porcentaje de materias primas importadas: resinas, pigmentos y lacas que no se producen actualmente en el país. El proceso productivo inicia con la extracción de los insumos del sector minero, como se observa en la Figura 1, todos los productos cerámicos se realizan combinando las materias primas plásticas, no plásticas y fundentes, moldeándolas y calentándolas a temperatura de cocción entre $1.200 C^{\underline{o}}$ y $1.500 C^{-o}$ (p.382). 


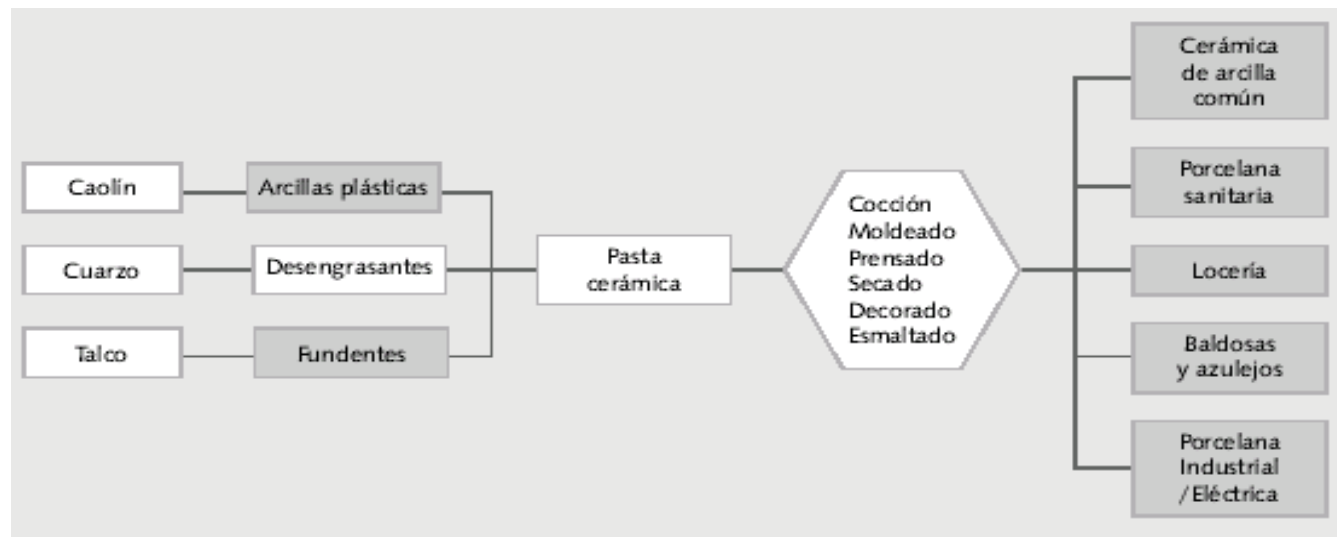

Figura 1

Cadena productiva de la cerámica

Fuente: Departamento Nacional de Planeación [DNP] (s.f, p.382)

En Colombia el sector cerámico y sus cadena productiva en los últimos años ha presentado un auge muy importante, reflejado en el aumento del nivel de las exportaciones de las empresas que desarrollan la fabricación y comercialización de estos productos; de igual manera, otra medida que ha contribuido al desarrollo del sector, son las políticas del gobierno nacional orientadas a estimular la construcción, ofreciendo créditos para la financiación de vivienda y subsidios que permiten generar una mayor demanda de los productos cerámicos, debido al crecimiento de la construcción en el país.

El PIB de este sector, como se observa en la Figura 2, en el año 2015 reflejó un desempeño superior al de la economía colombiana con una tasa del 4,6\%, aunque con una disminución en su crecimiento frente al año 2014, cuando alcanzó un crecimiento de 11,2 \% (Asociación de Industriales de Colombia, [ANDI] 2015). A pesar de esto al cierre del 2015, este sector sigue impulsando el crecimiento económico, con un aumento del 3,1 \% del total del Producto Interno Bruto. 


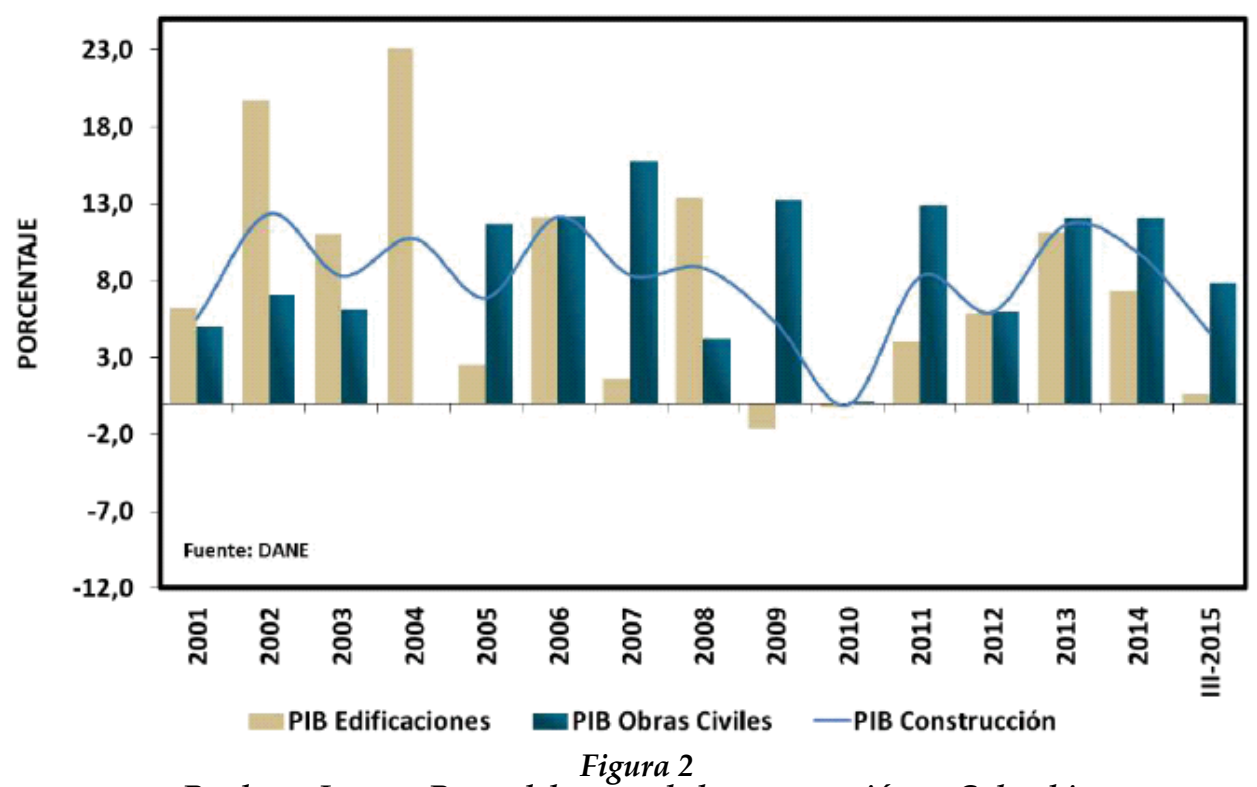

Producto Interno Bruto del sector de la construcción en Colombia Fuente: ANDI $(2015$, p.36)

En este sentido, el sector de la construcción es una actividad dinamizadora del sector cerámico; ya que este material representa uno de los principales insumos para la construcción, siendo las empresas de cerámica quienes fabrican y comercializan estos productos empleados para el revestimiento de pisos y paredes, así como los materiales de porcelana sanitaria.

Considerando esto, al observar los sectores que impulsan la comercialización de la cerámica, se encuentra que en términos de exportaciones de materiales de la construcción sumaron 475 millones de dólares en el 2015; concentrando un 70\% de sus ingresos en cinco países: Estados Unidos, Panamá, Venezuela, Perú y Ecuador, tal como lo muestra la Figura 3. 


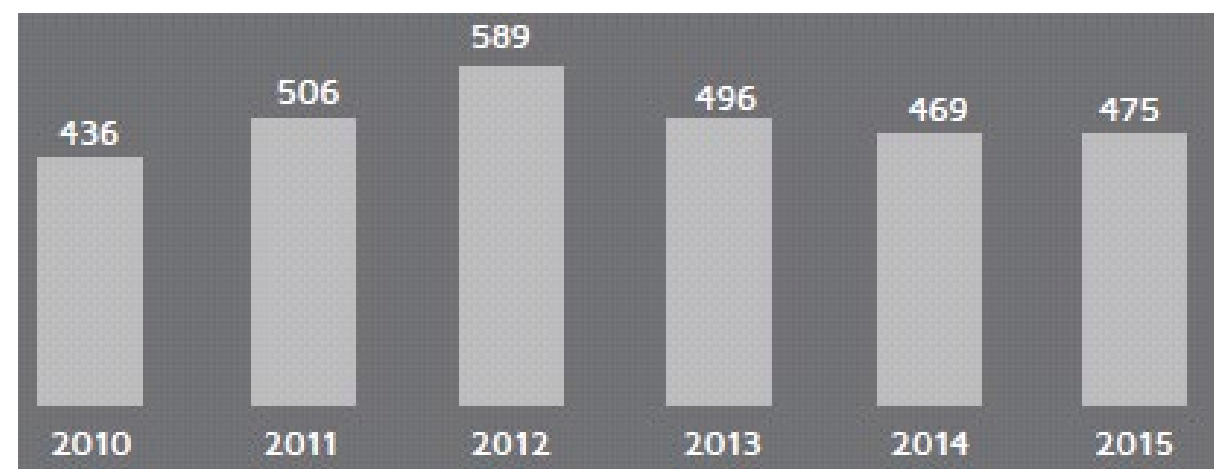

Figura 3

Exportaciones de materiales de construcción

Fuente: Procolombia (2016, p.4)

Es así como, en términos de crecimiento de económico, el consumo de materiales ha presentado un crecimiento que impulsa el avance con una dinámica positiva del 2010 al 2014 en un 6,7 \%, como lo refleja la Figura 4, según datos de Procolombia (2016).

En el departamento de Norte de Santander, la industria cerámica está conformada por empresas que fabrican productos no metálicos para la construcción, convirtiéndose en el departamento pionero en la producción y explotación de baldosas de cerámica, dado por la ventaja competitiva y comparativa de la arcilla en la región, beneficios del producto que favorecen comercial y financieramente las empresas de cerámica de Cúcuta, es por esto que se constituyen las principales generadoras de empleos directos e indirectos, favoreciendo el desarrollo económico y social de la región, de acuerdo a lo planteado por Sánchez \& Monroy (2008). Por otra parte, estas empresas son las principales generadoras de puestos de trabajo con aproximadamente 8.900 personas de las cuales el 92,64\% son hombres, quienes se desempeñan en el área de producción (Sánchez \& Monroy, 2008). 


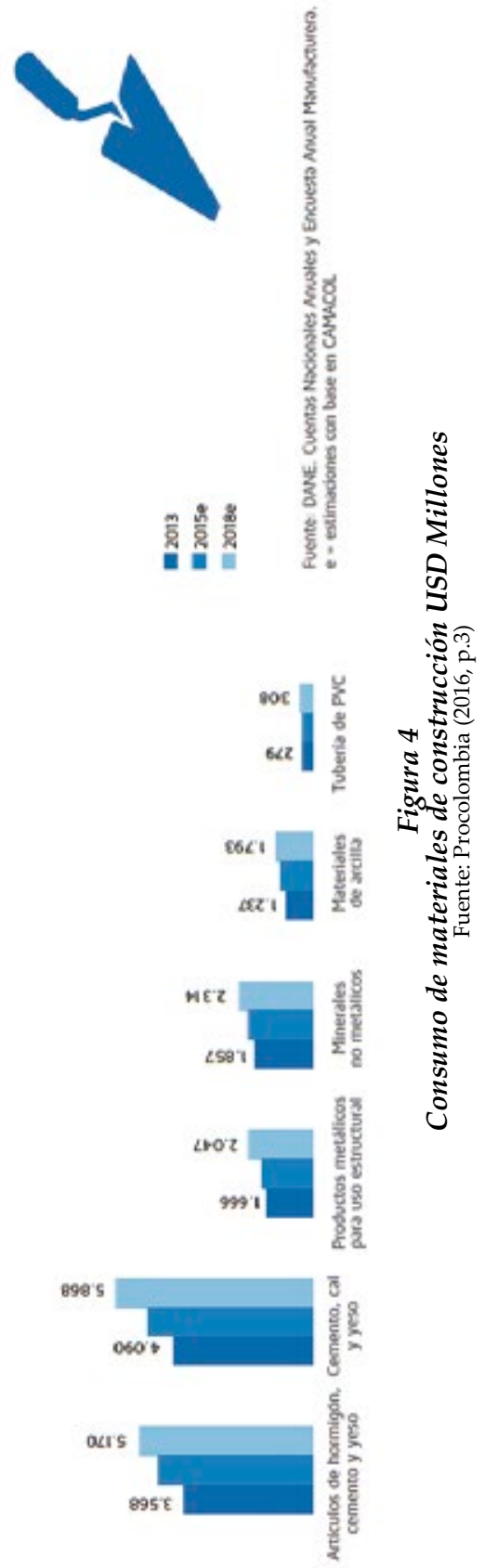


De manera, que si se observa a la empresa como una organización con en un entorno social (Torres-Salazar \& Melamed-Varela, 2016), "hay una sola responsabilidad social de las empresas, que no es más sino que la de utilización de sus recursos y comprometerse en actividades diseñadas para incrementar sus utilidades" (Robledo, 2006, p.13).

Es por esto de suma importancia conocer la situación financiera de las empresas, la revisión periódica y evaluación de su desempeño interno, enmarcado en un pleno conocimiento de la disponibilidad de sus recursos financieros en corto plazo, el estado y gestión de sus inventarios, cuentas por cobrar a clientes y flujo de caja libre con el objetivo de establecer estrategias que permitan implementar acciones de mejora y consolidar aquellos procesos que le son favorables y que conlleven a la generación de valor de la organización.

Con respecto a esto, los inventarios y las cuentas por cobrar constituyen importantes inversiones para la mayoría de las empresas; estos dos activos circulantes representan proporciones considerables de los activos totales, por consiguiente se puede decir que una adecuada administración de estos activos es esencial para el cumplimiento de los objetivos financieros en busca de la maximización de las riquezas y la creación de valor de las empresas.

Cuando se hace referencia al crédito que se otorga al cliente, Gitman (1996) opina: “Las cuentas por cobrar representan la extensión de un crédito a sus clientes en cuenta abierta, con el fin de mantener sus clientes habituales y atraer nuevos" (p.94). De aquí la importancia del empuje que le pueden dar al maximizar la empresa, ya que la correcta administración de las cuentas por cobrar contribuye al crecimiento de la empresa. 
Por otra parte, un inventario representa la existencia de bienes muebles e inmuebles que tiene la empresa para comerciar con ellos, comprándolos y vendiéndolos tal cual o procesándolos primero antes de venderlos, en un período económico determinado; deben aparecer en el grupo de activo circulante.

Según Chiavenato (1993), “Existencias es la composición de materiales que no se utilizan en el momento en la empresa, pero que necesitan existir en función de futuras necesidades"(p.26). Es decir, son aquellos productos o materias primas que la empresa dispone para el logro de su objeto social, que no son utilizadas en el momento que se adquieren, pero que son necesarios en un momento determinado.

Para realizar la caracterización del disponible, los inventarios, las cuentas por cobrar a clientes, los proveedores y las obligaciones financieras a corto plazo, se partió sobre el hecho que la liquidez es la capacidad que posee una empresa para generar los recursos necesarios para el cubrimiento de sus compromisos a corto plazo, tanto operativos como financieros, un sentido dinámico al análisis de la aplicación de recursos, mediante la comparación entre cuentas del balance estáticas y cuentas de resultados dinámicas.

Cuando se refiere a rentabilidad, García (2009) afirma que "La rentabilidad es la medida de la productividad de los fondos comprometidos en un negocio" (p.240). Es decir, es el resultado del proceso productivo y distributivo; es la utilidad o ganancia del negocio por realizar sus actividades. Mide el éxito de la empresa así como la suficiencia, siendo un indicador del rendimiento.

Tomando como referencia que la rentabilidad es un índice financiero que evalúa el beneficio del capital invertido ya sea por parte de los 
dueños de la empresa o los acreedores cuando conceden préstamos, en función del desarrollo del objeto social de la organización se ha propuesto en la investigación determinar ¿cuál fue la rentabilidad de las empresas de cerámica de Cúcuta?.

El flujo de caja libre, según García (2009), es el saldo disponible para pagar a los accionistas y para cubrir el servicio de la deuda (intereses de la deuda + principal de la deuda) de la empresa, después de descontar las inversiones realizadas en activos fijos y en necesidades operativas de fondos; es la medida financiera que muestra verdaderamente el valor de una organización; es decir, es la cantidad de flujo de efectivo en operación que permanece después de reemplazar la capacidad de producción actual y mantener los dividendos o participaciones de capital actuales.

El flujo de caja libre tiene dos objetivos básicos: cubrir el servicio de la deuda y cubrir el reparto de utilidades a los socios; es necesario para toda organización conocer su flujo de caja libre, ya que el crecimiento se asocia con demanda de efectivo, la participación de mercado con la generación de efectivo. El flujo de caja puede entonces generar y/o demandar efectivo; conocer esta medida financiera permite a la empresa tomar decisiones en cuanto a la expansión, ya que el crecimiento esperado del flujo de caja a perpetuidad determina el valor de una empresa.

En consonancia con la literatura investigada, se planteó encontrar el punto óptimo adecuado de nivel de cuentas por cobrar a clientes, efectivo, inventarios, proveedores, obligaciones financieras, la liquidez y el flujo de caja libre, desarrollando un análisis y una adecuada gestión y toma de decisiones financieras que generen valor en la organización. 


\section{MÉTODO}

Se recurrió al análisis cuantitativo y a la revisión e interpretación de datos secundarios. El nivel fue descriptivo correlacional y la unidad de análisis estuvo constituida por la información contable y financiera de la empresa Cerámica Italia S.A. Para el cálculo de la rotación de cartera, rotación de inventario, comportamiento de las cuentas del efectivo, cuentas por cobrar, proveedores, obligaciones financieras y flujo de caja libre se siguió la metodología propuesta por García (2009).

\section{RESULTADOS, DISCUSIONES Y CONCLUSIONES}

Al investigar la estructura de la liquidez del sector cerámico en los años de estudio se observó un aumento de las cuentas por cobrar a clientes e inventarios, afectando el retorno del efectivo sobre la inversión realizada, provocando fondos ociosos improductivos por la acumulación de inventario y la ineficiencia del recaudo de la cartera, y como consecuencia impactando "la capacidad que tiene la empresa de generar los fondos suficientes para el cubrimiento de sus compromisos de corto plazo, tanto operativos como financieros" García (2013, p.15).

Por otra parte y teniendo en cuenta que el flujo de caja libre permite determinar el nivel de efectivo disponible para atender a los compromisos de caja, se analizó el comportamiento de este en los años de estudio, observando que aumentó año a año, por la disminución de la inversión del capital de trabajo y la reposición de activos fijos y no por la generación de recursos propios, dado que el nivel de liquidez se redujo por la ineficiencia en la gestión operacional en los años de estudio.

Como resultado de la investigación se presenta a continuación la caracterización de cada una de las variables investigadas y la correlación 
con el flujo de caja libre y generación de valor en el sector cerámico.

\section{Caracterización de las cuentas por cobrar a clientes años 2005-2015}

Los créditos en una empresa por la comercialización de sus productos representan la financiación que se otorga al cliente de acuerdo a las políticas financieras de financiación y liquidez de una organización, estas son el crédito comercial que la empresa extiende a otras organizaciones o consumidores finales, siendo el mantenimiento de estas partidas del balance general la representación sustancial de un costo por el valor del dinero en el tiempo, al no recaudar el dinero o un beneficio para la organización por el aumento de sus ingresos por venta de sus productos; es por esto que el diseño y manejo de políticas de crédito, el cobro adecuado de la cartera y la gestión de estas cuentas son algunos de los aspectos más importantes y de mayor impacto en el desempeño general de la compañía.

Acorde a la anterior premisa, al realizar el análisis del comportamiento de las cuentas por cobrar a clientes y la gestión de estas en la empresa Cerámica Italia S.A, se observó cómo presentaron un incremento año a año, con una leve desaceleración del $9 \%$ en el periodo comprendido del año 2009 al 2010, que no fue constante en los periodos siguientes, y que por el contrario, aumentó de forma constante, comprometiendo la liquidez de la empresa.

En este sentido, con respecto a la inversión en activos corrientes, debido a la convertibilidad en efectivo que representa el cobro de las cuentas por cobrar a clientes en la organización, se encuentra que la empresa a medida que aumenta su inversión en activos corrientes, aumenta las cuentas por cobrar a clientes, comprometiendo año a año la liquidez de la compañía, tal como se observa en la Figura 6. 


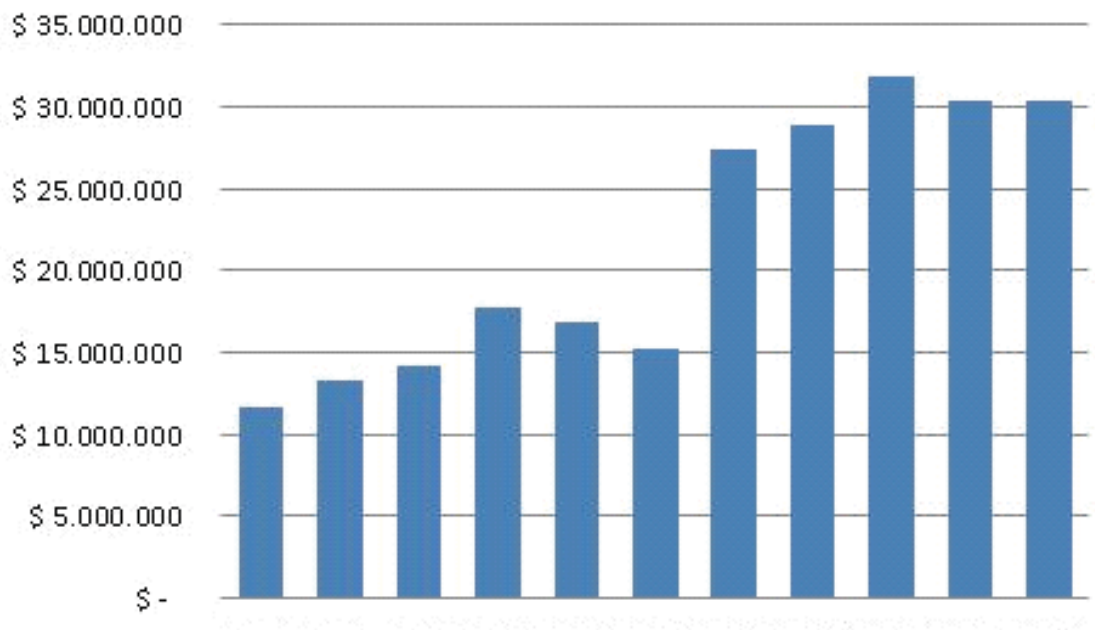

20052006200720082009201020112012201320142015

- CUENTAS POR COBRAR A CLIENTES

Figura 5

Comportamiento de las cuentas por cobrar 2005-2015

Fuente: Elaboración propia

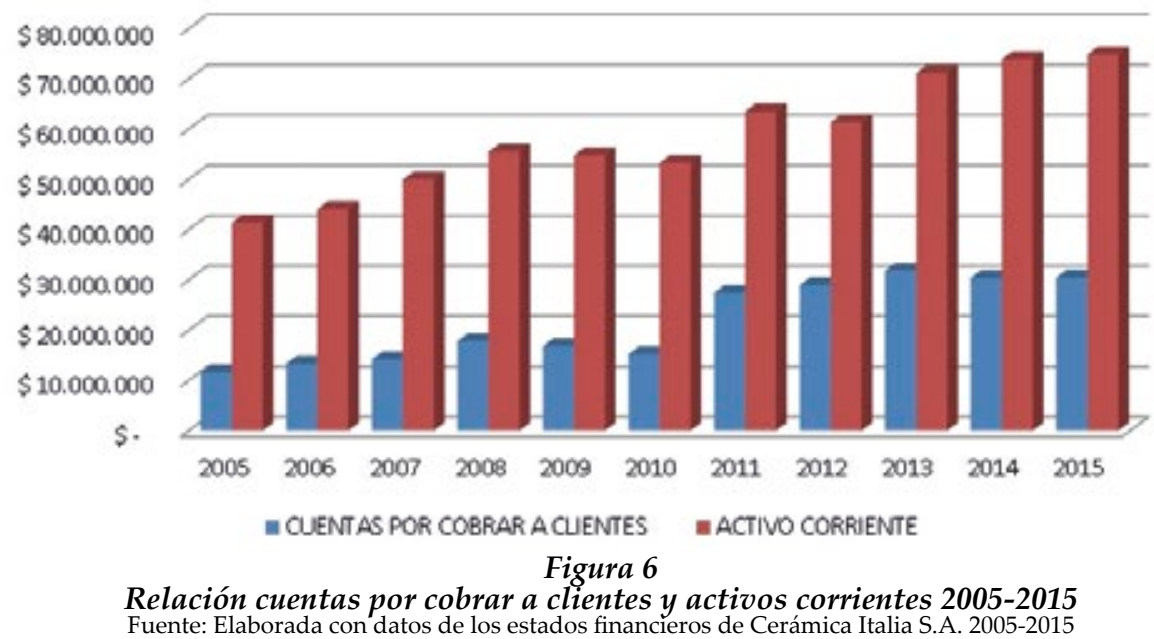

La rotación de cuentas por cobrar, según Ortiz (2009), es el indicador mide el número de veces en que giran las cuentas por cobrar, es decir, el periodo de tiempo que se demora la empresa para convertir en efectivo las ventas. 
A continuación se puede observar en la Figura 6, que la rotación de cuentas por cobrar a través de los años en la empresa Cerámica Italia se ha visto afectada haciendo que el proceso de recaudo sea más lento de un año a otro; con periodos cortos de recuperación que generan un mejor recaudo de la cartera, como se observa del año 2009 al 2010 y del 2014 al 2015; sin embargo, la empresa presenta periodos de recuperación del dinero dado en crédito a los clientes, lo que compromete liquidez y rentabilidad.

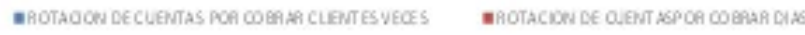

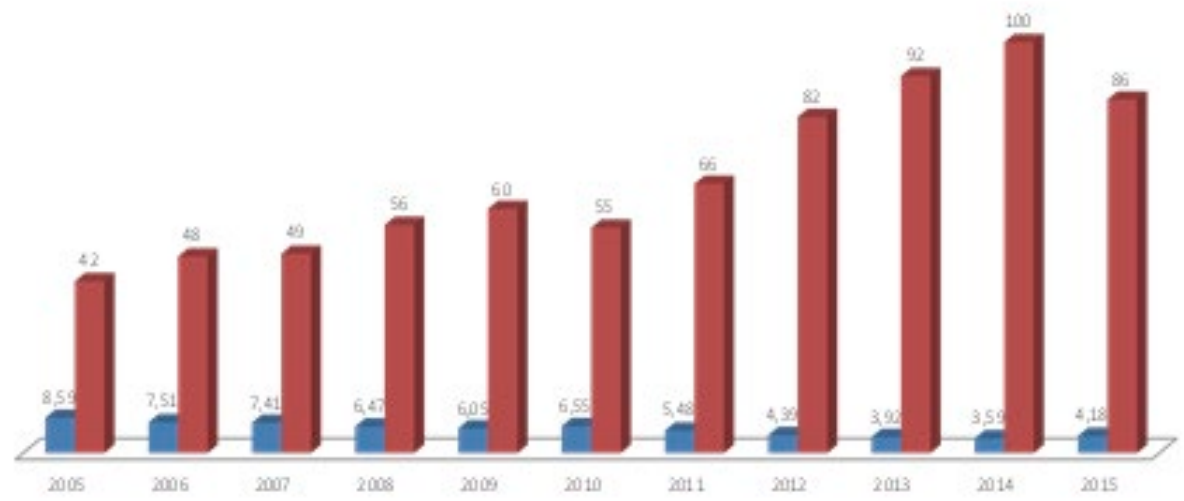

Figura 7

Comportamiento de la rotación de las cuentas por cobrar en veces y en días 2005-2015

Fuente: Elaborada por los autores con datos de los estados financieros de Cerámica Italia S.A. 2005-2015

De esta manera y al observar la escasa recuperación del dinero que representa las ventas por créditos otorgados a los clientes, se puede evidenciar que las ventas del año 2005 al 2015 han crecido, pero este crecimiento ha ido desacelerando en el tiempo, aumentando en menor proporción y solo disminuyendo del año 2013 al 2014 del 3,81 \% y 6,11 \%. Sin embargo, en el año 2015 la empresa presentó una recuperación sustancial de los ingresos por ventas, con un aumento de 13,56 \%. 
Al comparar este comportamiento con el de las cuentas por cobrar, se observó que las ventas están aumentando en menor proporción que las cuentas por cobrar, lo que reflejó que no se ejecutaron de una forma adecuada las políticas de cuentas por cobrar, como se observa en la Figura 8.

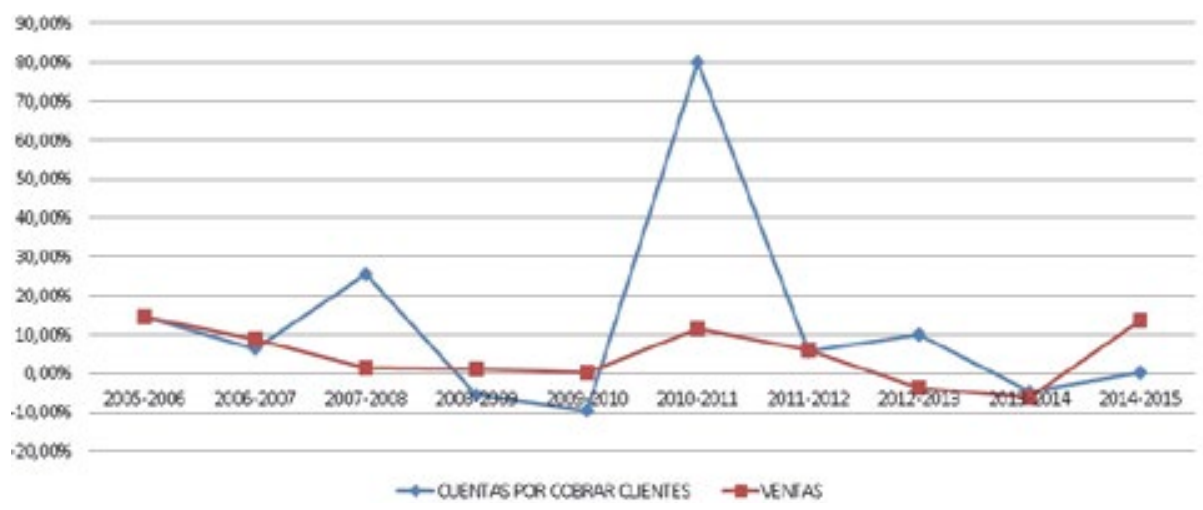

Figura 8

Comportamiento de las cuentas por cobrar y las ventas 2005-2015

Fuente: Elaborado con datos de los estados financieros de Cerámica Italia S.A. 2005-2015

\section{Caracterización de los inventarios años 2005-2015}

Los inventarios de materias primas, productos en proceso y productos terminados constituyen el almacenamiento de las mercancías disponibles para la producción y venta, con el objetivo de satisfacer las necesidades de los clientes, siendo esenciales para la consecución de recursos económicos, ventas y utilidades; es por esto que su control es muy importante para la empresa, ya que del seguimiento y monitoreo que se establezca, depende que los costos de estos inventarios sean más bajos, por ello mantener un excesivo inventario puede arruinar a una compañía por causa de fondos ociosos improductivos, a su vez los faltantes también pueden ser tan dañinos como los excesos.

La empresa Cerámica Italia S.A. organización dedicada a la produc- 
ción de materiales para la construcción, cuenta con un inventario de materias primas, productos en proceso y productos terminados.

En la estructura de sus activos la inversión en inventarios represento una participación entre el $20 \%$ y 37 \%; observando un incremento del $75 \%$ en la concentración de estos activos del año 2005 al 2006 y una leve disminución de 13 \% y 1 \% en los años 2009 y 2012.

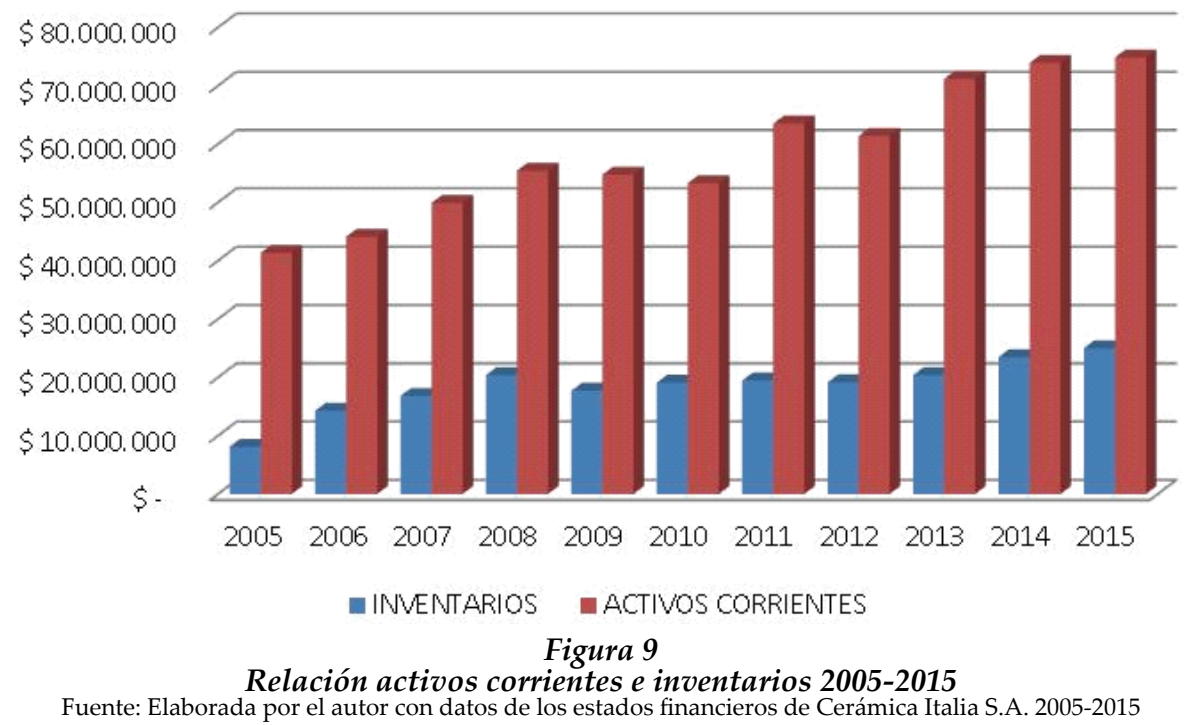

Este comportamiento de los inventarios y su incremento posiblemente corresponda a una respuesta de la administración de la empresa al observar del año 2003 al 2005 un aumento aproximado del $20 \%$ en las exportaciones del país (ver Figura 10). 


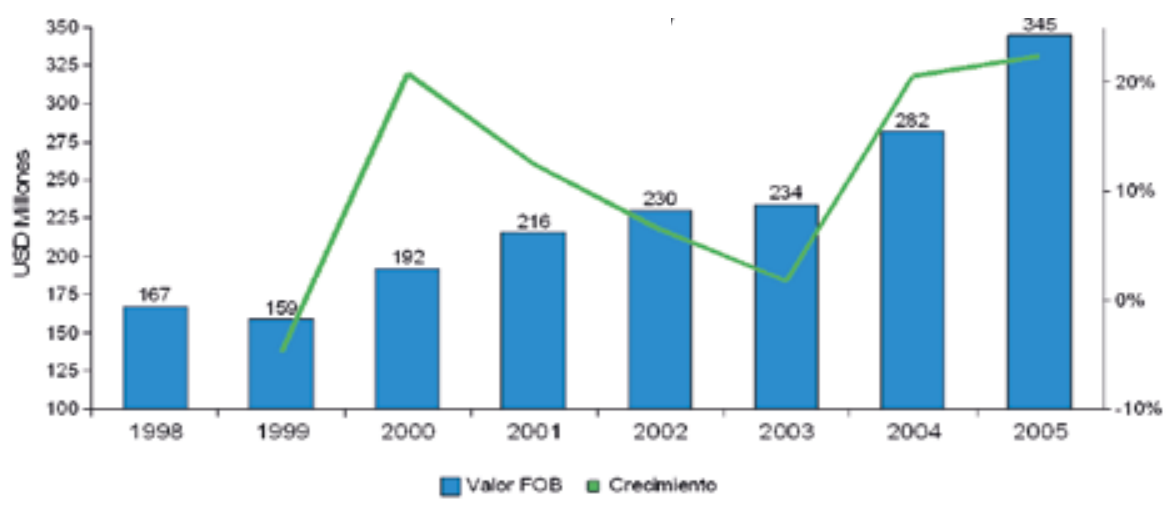

Figura 10

Exportaciones de materiales de la construcción 1999-2005

Fuente: Proexport (2008)

En este sentido, se analizó el comportamiento de las ventas, el costo y los niveles de inventarios de la empresa Cerámica Italia S.A. en 20052015, encontrando que los niveles de inventario en la mayor parte del periodo estudiado aumentaron en mayor proporción de lo que lo hicieron las ventas, el nivel que invirtió la empresa, su efectivo en inventarios, no se ha reflejado en ventas en los años posteriores a la inversión.

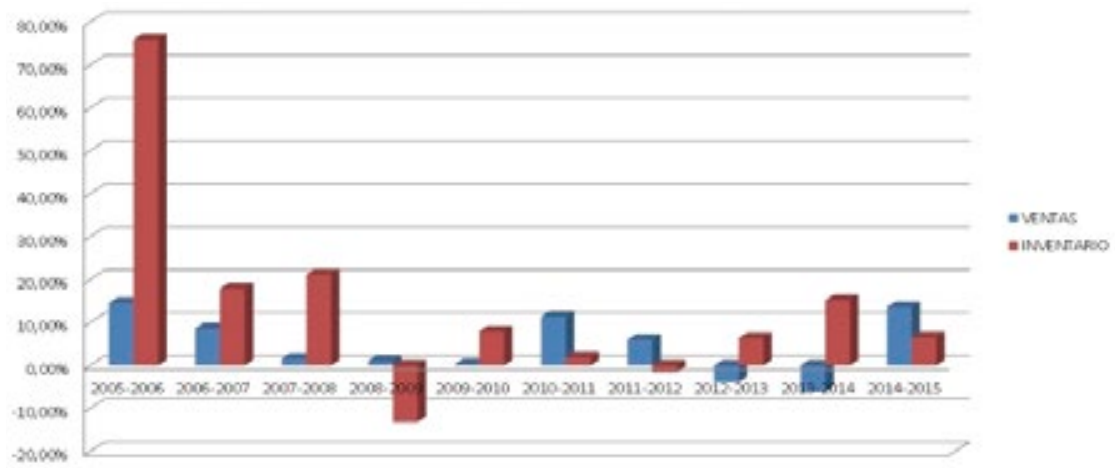

Figura 11

Comportamiento de las ventas y los inventarios 2005-2015

Fuente: Elaborado con datos de los estados financieros de Cerámica Italia S.A. 2005-2015

Para medir la eficiencia de los inventarios en cuanto a la recuperación de efectivo invertido se calcularon los indicadores de rotación de inventarios. 
Se observa que la empresa tiene unos niveles de inventarios muy altos, también es preciso decir que del año 2005 al 2015 ha disminuido la rotación de los inventarios ocasionando como consecuencia que se haga más lenta la salida del inventario existente; lo que puede ocasionar recursos improductivos por el mal manejo del inventario (procesos de compra y mantenimiento del mismo) o porque no se generan las ventas necesarias para que el inventario rote.

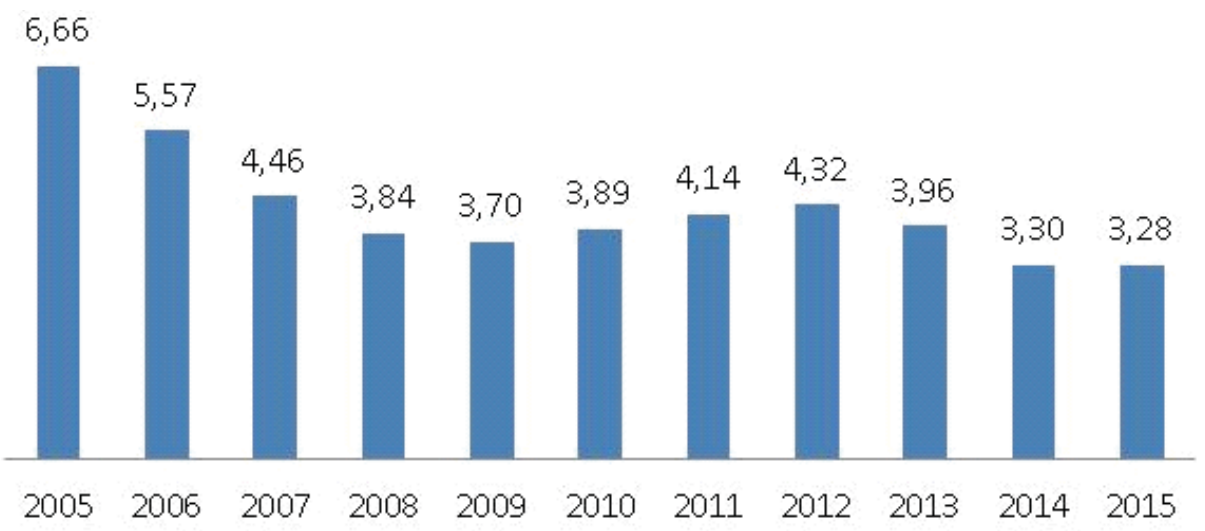

Figura 12

Comportamiento de la rotación de inventarios 2005-2015

Fuente: Elaborado con datos de los estados financieros de Cerámica Italia S.A. 2005-2015

Es importante resaltar que a partir del año 2006 el sector edificador ha reducido el ritmo de expansión que había reflejado en los últimos años como consecuencia de la desaceleración de la economía nacional y por el menor crecimiento mundial.

Debido a que las cuentas por cobrar y los inventarios aumentaron en mayor proporción que las ventas se puede decir que la empresa está acumulando fondos ociosos o improductivos, ya que este exceso está ocasionando un costo de oportunidad a la empresa que se pudo haber utilizado para reducir deuda financiera o reparto de dividendos a los accionistas. 


\section{Caracterización del flujo de caja libre}

El flujo de caja libre es el saldo disponible para pagar a los accionistas y para cubrir el servicio de la deuda (intereses de la deuda + principal de la deuda) de la empresa, después de descontar las inversiones realizadas en activos fijos y en necesidades operativas de fondos; es la medida financiera que muestra verdaderamente el valor de una organización; es decir, es la cantidad de flujo de efectivo en operación que permanece después de reemplazar la capacidad de producción actual y mantener los dividendos o participaciones de capital actuales.

El flujo de caja libre tiene dos objetivos básicos: cubrir el servicio de la deuda y cubrir el reparto de utilidades a los socios; es necesario para toda organización conocer su flujo de caja libre, ya que el crecimiento se asocia con demanda de efectivo, la participación de mercado con la generación de efectivo. El flujo de caja puede entonces generar y/o demandar efectivo; conocer esta medida financiera permite a la empresa tomar decisiones en cuanto a la expansión, ya que el crecimiento esperado del flujo de caja a perpetuidad determina el valor de una empresa. 


\begin{tabular}{|c|c|c|c|c|c|c|c|c|c|c|c|c|c|c|}
\hline 율 & 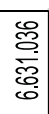 & 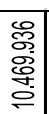 & 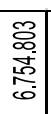 & 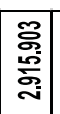 & 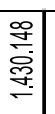 & 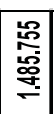 & 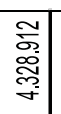 & 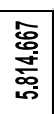 & 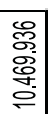 & 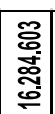 & 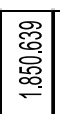 & \begin{tabular}{l} 
总 \\
察 \\
\multirow{2}{*}{}
\end{tabular} & $\begin{array}{l}\overline{\tilde{m}} \\
\text { ơ⿱ } \\
\text { ơ }\end{array}$ & 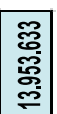 \\
\hline 퐁 & 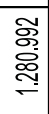 & 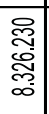 & 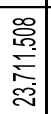 & 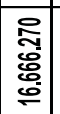 & 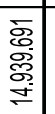 & 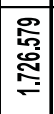 & 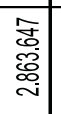 & \begin{tabular}{l}
\multirow{3}{*}{} \\
品
\end{tabular} & 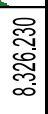 & 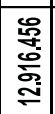 & 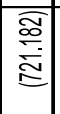 & 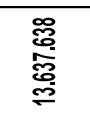 & 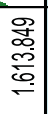 & 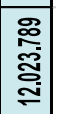 \\
\hline m্ㄱ & 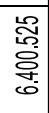 & 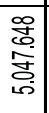 & 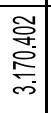 & 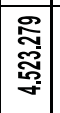 & 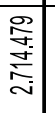 & \begin{tabular}{|l|} 
怘 \\
o. \\
o. \\
\end{tabular} & 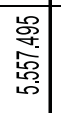 & 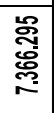 & 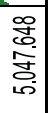 & 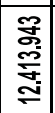 & \begin{tabular}{|l|}
\multirow{2}{\sigma}{} \\
o. \\
d. \\
\end{tabular} & 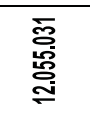 & 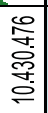 & 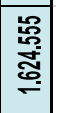 \\
\hline స్ & 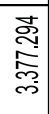 & 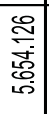 & 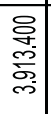 & 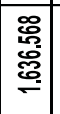 & 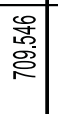 & $\begin{array}{l}\text { శ్ } \\
\text { స్ }\end{array}$ & 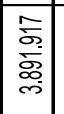 & 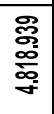 & 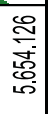 & 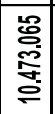 & 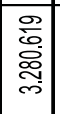 & $\begin{array}{l}\text { 导 } \\
\text { 曽 }\end{array}$ & 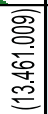 & 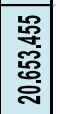 \\
\hline 5 & 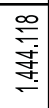 & 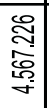 & 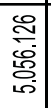 & 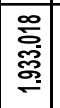 & 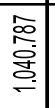 & 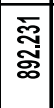 & 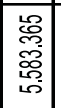 & 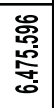 & 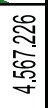 & 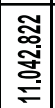 & 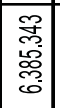 & 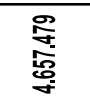 & 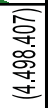 & 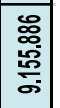 \\
\hline 3 & 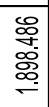 & 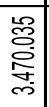 & 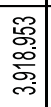 & 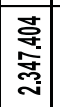 & 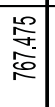 & 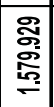 & \begin{tabular}{l|l}
$\dddot{8}$ \\
$\$$ \\
0 \\
0 \\
0 \\
0
\end{tabular} & 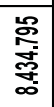 & 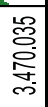 & 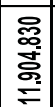 & 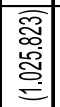 & 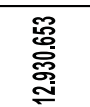 & 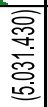 & 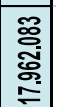 \\
\hline 8 & 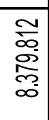 & 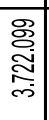 & 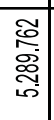 & 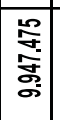 & 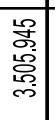 & 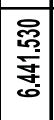 & 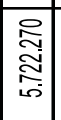 & 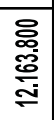 & 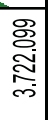 & 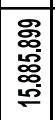 & \begin{tabular}{|l|}
$\widetilde{\delta}$ \\
。్ల \\
\end{tabular} & 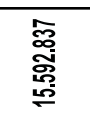 & 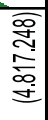 & 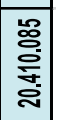 \\
\hline 骂 & 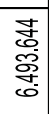 & 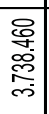 & 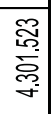 & 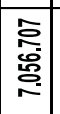 & 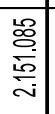 & 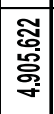 & 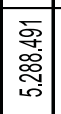 & $\begin{array}{l}\stackrel{m}{+} \\
\stackrel{9}{0} \\
\stackrel{0}{0}\end{array}$ & 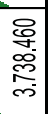 & 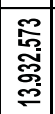 & 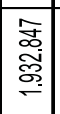 & 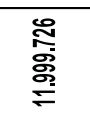 & $\begin{array}{l}\text { 웅 } \\
\text { 总 } \\
\stackrel{6}{=}\end{array}$ & 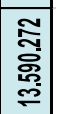 \\
\hline న్ & 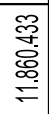 & 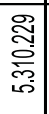 & 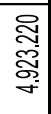 & 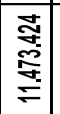 & 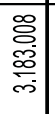 & 电 & \begin{tabular}{|l|} 
\\
\\
0 \\
0 \\
0 \\
$\sigma$ \\
\end{tabular} & 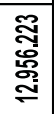 & 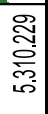 & 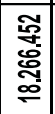 & 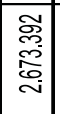 & 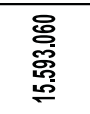 & $\begin{array}{l}\frac{1}{2} \\
\frac{5}{8} \\
\stackrel{\rho}{m}\end{array}$ & 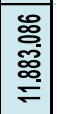 \\
\hline 总 & 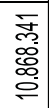 & 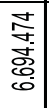 & $\underset{\text { is }}{\stackrel{P}{\rightleftarrows}}$ & $\begin{array}{l}\text { 商 } \\
\text { o. } \\
\text { of }\end{array}$ & 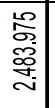 & 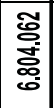 & 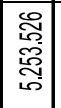 & 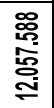 & 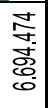 & 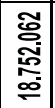 & 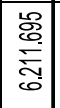 & 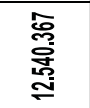 & 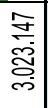 & 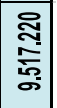 \\
\hline 总 & 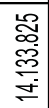 & 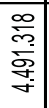 & 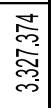 & 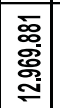 & 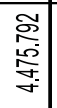 & 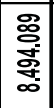 & $\begin{array}{l}\underset{\widetilde{\Xi}}{\tilde{\sigma}} \\
\underset{\sim}{\tilde{N}} \\
\end{array}$ & 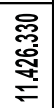 & $\begin{array}{l}\frac{\infty}{\infty} \\
\stackrel{\infty}{-} \\
\stackrel{\sigma}{\sigma} \\
\end{array}$ & 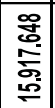 & 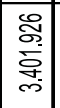 & 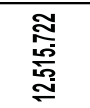 & 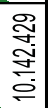 & 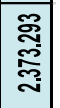 \\
\hline & 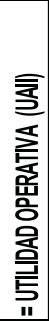 & 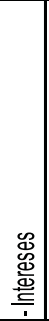 & 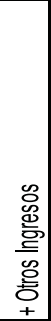 & 颉 & 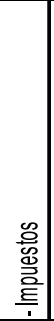 & 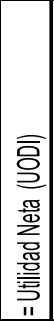 & 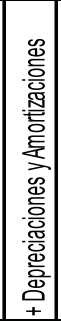 & 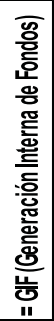 & 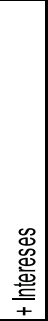 & 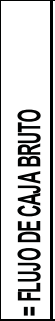 & 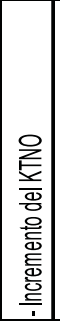 & 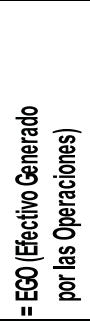 & 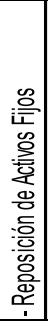 & 린 \\
\hline
\end{tabular}


La Generación Interna de Fondos (GIF) es parte del flujo de caja producido por la empresa que se genera en la misma, que se retiene para cubrir las inversiones no estratégicas, abonar al capital de la deuda, repartir utilidades, apoyar las inversiones estratégicas; es la fuente más importante, ya que representa los fondos que directamente se producen en la operación del negocio y da la pauta para evaluar las posibilidades a largo plazo de la empresa asociadas principalmente con el crecimiento, el reparto de dividendos y el pago de deuda financiera.

En el periodo estudiado de la empresa Cerámica Italia S.A ha presentado una disminución año a año; si observamos este comportamiento se puede decir que del año 2005 al 2015 ha disminuido el crecimiento de $\$ 11.426 .330$ del 2005 a $\$ 5.814 .667$, es decir, $\$ 5.611 .663$ menos, que pierde la empresa en la generación de fondos propios; generados por la disminución de la utilidad operativa y el aumento en la inversión de los activos.

En cuanto al flujo de caja bruto es el flujo de caja que a la empresa le queda disponible para cubrir las inversiones no estratégicas (reposición de capital de trabajo y activos fijos), atender el servicio a la deuda (capital más intereses), repartir utilidades, apoyar las inversiones estratégicas, la empresa presentó aumentos y disminuciones en los años estudiados; en cuanto al Efectivo Generado por Operaciones (EGO) es el flujo de caja que a la empresa le queda disponible para cubrir las inversiones no-estratégicas en activos fijos (reposición), atender el servicio a la deuda (capital más intereses), repartir utilidades, apoyar las inversiones estratégicas, en la empresa Cerámica Italia S.A. También se observó aumento y disminuciones dados por la disminución o incremento de la reposición del capital de trabajo neto operativo. 
Por otra parte, el flujo de caja libre del año 2005 al 2015 ha tenido periodos de constantes aumentos, determinados básicamente por las decisiones financieras tomadas con respecto a la reposición de activos fijos, observando la recuperación del flujo de caja libre por la liberación de activos fijos o la mínima inversión realizada.

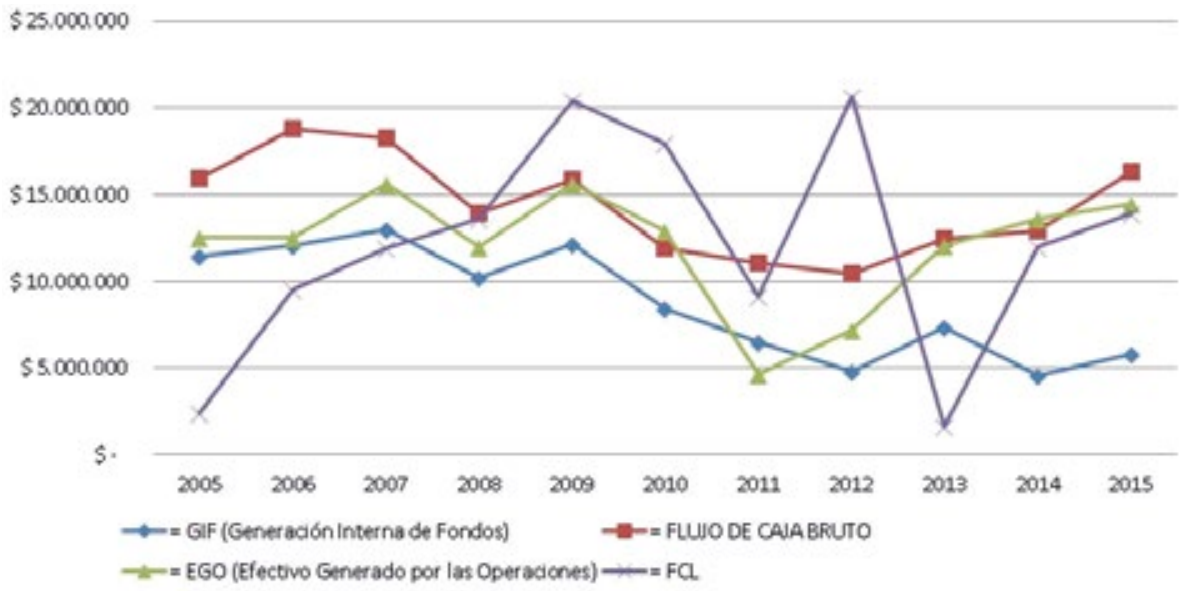

Figura 13

Flujo de caja libre 2005-2015

Fuente: Elaborado con datos de los estados financieros de Cerámica Italia S.A. 2005-2015

\section{Correlación de rotación de cuentas por cobrar y flujo de caja libre}

$\mathrm{Al}$ relacionar las cuentas por cobrar con todos los componentes del flujo de caja libre, tales como generación interna de fondos, flujo de caja bruto, efectivo generado por operaciones, presentan correlaciones positivas, quiere decir que estas variables se comportan en términos generales en igual sentido a la rotación de las cuentas por cobrar; en cuanto al flujo de caja libre y la relación de las cuentas por cobrar presentan correlación positiva. 


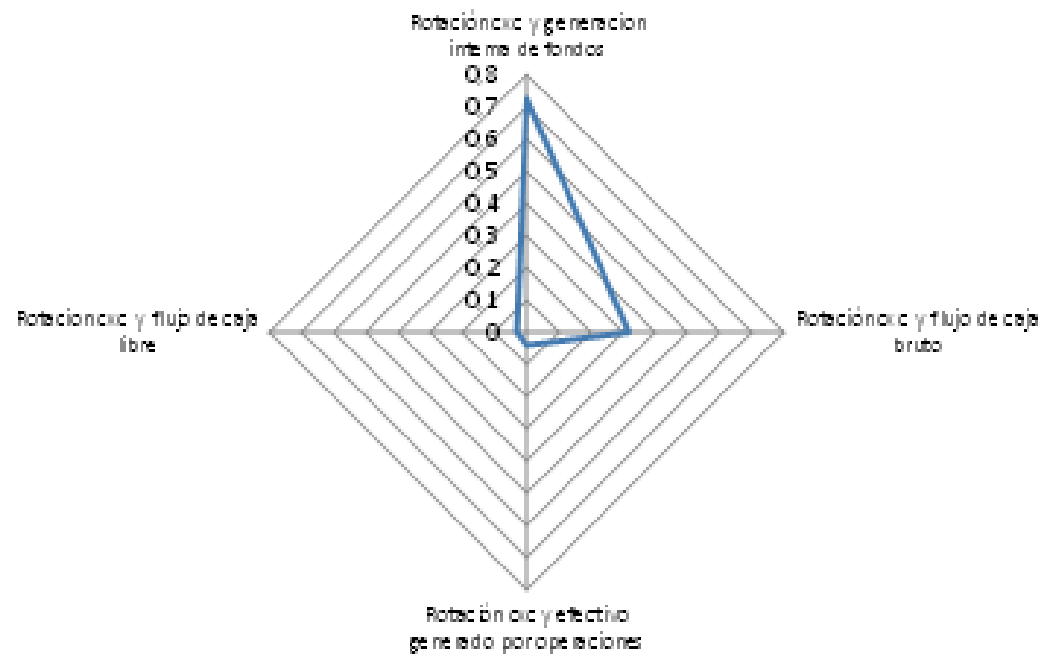

Figura 14

Correlación rotación de cuentas por cobrar, generación interna de fondos, flujo de caja bruto, efectivo generado por operaciones y flujo de caja libre

Fuente: Elaborado con datos de los estados financieros de Cerámica Italia S.A. 2005-2015

\section{Correlación de los inventarios con flujo de caja libre}

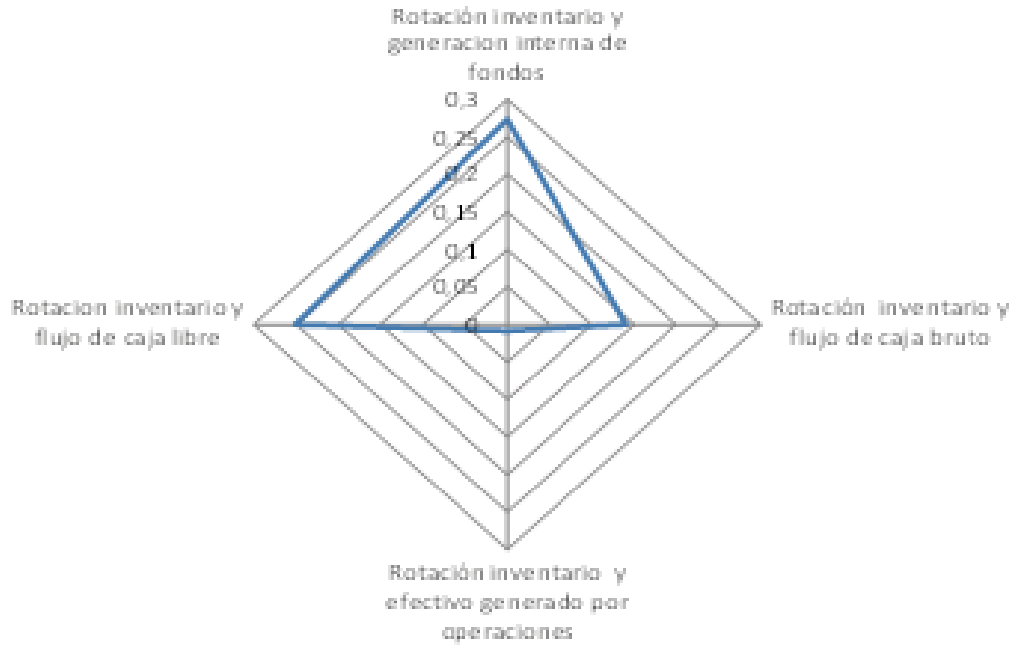

Figura 15

Correlación rotación de inventarios, generación interna de fondos, flujo de caja bruto, efectivo generado por operaciones y flujo de caja libre

Fuente: Elaborado con datos de los estados financieros de Cerámica Italia S.A. 2005-2015 
Los componentes del flujo de caja libre, tales como generación interna de fondos, flujo de caja bruto y efectivo generado por operaciones y flujo de caja libre, presentan correlaciones con respecto a la rotación de inventarios; quiere decir que se comportan en términos generales en igual sentido a la rotación de los inventarios.

En cuanto a los inventarios, se observa que la empresa tiene unos niveles muy altos, por otra parte, la rotación de los inventarios causa como consecuencia que se haga más lenta su salida; lo que puede ocasionar recursos improductivos por el mal manejo de procesos de compra y mantenimiento del mismo o porque no se generan las ventas necesarias para que el inventario rote; igualmente, también se denota que el costo de ventas ha aumentado en un porcentaje mayor a las ventas.

Tabla 2

Matriz de gestión de estrategias financieras para mejorar la gestión de cuentas por cobrar, inventarios, rentabilidad y flujo de caja libre

\begin{tabular}{l|l|l|l}
\hline $\begin{array}{l}\text { VARIABLE DE } \\
\text { DESEMPEÑO }\end{array}$ & \multicolumn{1}{|c|}{$\begin{array}{c}\text { ESTRATEGIAS } \\
\text { FINANCIERAS }\end{array}$} & \multicolumn{1}{c}{$\begin{array}{c}\text { OBJETIVOS } \\
\text { ESTRATÉGICOS }\end{array}$} & \multicolumn{1}{c}{$\begin{array}{c}\text { ACCIONES } \\
\text { ESTRATÉGICAS }\end{array}$} \\
\hline $\begin{array}{l}\text { Cuentas por } \\
\text { cobrar }\end{array}$ & $\begin{array}{l}\text { Reducción de cuentas } \\
\text { por cobrar. } \\
\text { Aumento en la rotación } \\
\text { de cartera. }\end{array}$ & $\begin{array}{l}\text { Aumentar las ventas en } \\
\text { efectivo. } \\
\text { Ejercer políticas de } \\
\text { cobranza adecuadas. }\end{array}$ & $\begin{array}{l}\text { Establecimiento de un plan co- } \\
\text { mercial que aumente las ventas } \\
\text { en efectivo. } \\
\text { Diseño de un manual de cré- } \\
\text { dito, donde se establezcan } \\
\text { condiciones, forma de pago y } \\
\text { recaudo de cartera. }\end{array}$ \\
$\begin{array}{l}\text { Inventarios } \\
\text { Reducción de los } \\
\text { inventarios. } \\
\text { Aumento de la rotación } \\
\text { de inventarios. }\end{array}$ & $\begin{array}{l}\text { Aumento de las ventas. } \\
\text { cación y evaluación del } \\
\text { inventario. }\end{array}$ & $\begin{array}{l}\text { Realizar un manual de adminis- } \\
\text { tración y control de inventario, } \\
\text { donde se evalúe, costos y } \\
\text { necesidades de ventas. }\end{array}$ \\
$\begin{array}{l}\text { Flujo de caja } \\
\text { libre }\end{array}$ & $\begin{array}{l}\text { Aumento del flujo de } \\
\text { caja libre. }\end{array}$ & $\begin{array}{l}\text { Aumento del margen del } \\
\text { (EBITDA)* } \\
\text { Disminución de inversión } \\
\text { de capital de trabajo y re- } \\
\text { posición de activos fijos. }\end{array}$ & $\begin{array}{l}\text { Realizar una adecuada inver- } \\
\text { sión de capital de trabajo y } \\
\text { reposición de activos fijos. }\end{array}$ \\
\hline
\end{tabular}

* EBITDA: Beneficio antes de intereses, Impuestos, Depreciaciones y Amortizaciones (por sus siglas en inglés, Earnings, Before Interest Taxes, Depreciation and Amartization)

Fuente: Elaboración propia (2016) 
En los últimos años la empresa ha incrementado peligrosamente sus días de inventario; por este concepto se ha producido una destrucción de valor como consecuencia de la acumulación de fondos ociosos que implica esta situación.

Debido a que las cuentas por cobrar y los inventarios aumentaron en mayor proporción que las ventas la empresa está acumulando fondos ociosos o improductivos, ya que este exceso está ocasionando unos costos de oportunidad a la empresa que se pudieron haber utilizado para reducir deuda financiera o reparto de dividendos a los accionistas.

Es necesario determinar un plan de acción que plantee los pasos a seguir en cuanto al mejoramiento de las ventas y el recaudo de cartera, que a su vez propicie los atrasos que hay con los proveedores; lo que hace pensar que la empresa no cuenta con la liquidez necesaria para cancelar sus cuentas, para lo cual ha liberado activos fijos.

Para que Cerámica Italia S.A. encamine la toma de decisiones financieras de manera objetiva se hace necesario que la administración y la Junta Directiva, revisen el comportamiento de las variables de cuentas por cobrar, inventarios, rentabilidad y flujo de caja libre, a través de la elaboración de informes periódicos por parte de los profesionales de las distintas áreas competentes que permitan realizar mediciones permanentes y por ende, monitorear el comportamiento financiero.

\section{REFERENCIAS BIBLIOGRÁFICAS}

ANDI (2015). Colombia: Balance 2015 y Perspectivas 2016. Bogotá D.C., Colombia: Autor.

Chiavenato, I. (1993). Iniciación a la administración de materiales. Ciudad de México, México: McGraw-Hill. 
Departamento Nacional de Planeación (2006). Céramica. Recuperado de: https://colaboracion.dnp.gov.co/cdt/desarrollo\%20empresarial/ ceramica.pdf

García, D. (2013) Evaluación Financiera en los procesos de Contratación Estatal en Colombia. Repositorio Universidad Militar Nueva Granada, 1-21.

García, O. (2009). Administración Financiera Fundamentos y Aplicaciones (cuarta edición). Bogotá D.C., Colombia: Bernalibros S.A.S.

Gitman, L. (1996). Fundamentos de administración financiera (tercera edición). Ciudad de México, México: Harla.

Grupo Bancolombia (2007). Ventas del sector cerámico.

Ortiz, H. (2009). Análisis Financiero Aplicado: y principios de administración financiera. Bogotá D.C., Colombia: Ediciones Universidad Externado de Colombia.

Procolombia (2016). El mundo invierte en Colombia, inversión en materiales de construcción.

Proexport Colombia (2008). Materiales de construcción, macrosector manufacturas e insumos.

Robledo, C. J. (2006). El TLC recoloniza a Colombia (primera edición). Manizales, Colombia: Editores S.A.

Sánchez, J. y Monroy, R. (2008). Clúster de la cerámica en Norte de Santander, una opción para el desarrollo regional (Trabajo de Grado). Cúcuta, Colombia: Universidad Francisco de Paula Santander.

Torres-Salazar, P \& Melamed-Varela, E. (2016). Fundamentos teóricos de la cultura desde la óptica de las organizaciones. Desarrollo Gerencial, 8(1), 143-164. DOI: https://doi.org/10.17081/dege.8.1.1411 - Universidad Simón Bolívar. Barranquilla-Colombia.

Cómo citar este capítulo:

Jácome-Castilla, N. \& Sepúlveda-Angarita, M. (2017). Liquidez y el flujo de caja libre, Valoración de las empresas de cerámica de Cúcuta En Prieto-Pulido, R. \& Palacios-Arrieta, A. (2017). Gestión contable y financiera en las organizaciones. Un enfoque global. Ediciones Universidad Simón Bolívar. Barranquilla-Colombia. 153-179. 